\title{
Guest Editorial: MLSP 2020 Special Issue
}

\author{
Simo Särkkä ${ }^{1}$. Lassi Roininen ${ }^{2} \cdot$ Manon Kok $^{3} \cdot$ Roland Hostettler $^{4} \cdot$ Andreas Hauptmann $^{5,6}$
}

Accepted: 28 December 2021 / Published online: 6 January 2022

(c) The Author(s), under exclusive licence to Springer Science+Business Media, LLC, part of Springer Nature 2021

The topic of the 2020 IEEE International Workshop on Machine Learning (MLSP 2020), which was the 30th workshop in the series, was at the intersection of Machine Learning (ML) and Signal Processing (SP). Although both ML and SP are important areas as such, their combination provides an even more valuable framework which can be, for example, used in so-called artificial intelligence (AI) systems. The workshop itself was held during a global pandemic caused by COVID-19 and was therefore held fully online. Despite this, the scientific quality of the workshop was great.

This special issue consists of extended versions of a selected subset of papers published in the proceedings of MLSP 2020. The papers to the special issue where invited among the papers that got the highest ratings from the reviewers of the workshop. All the papers went though a

Simo Särkkä

simo.sarkka@aalto.fi

Lassi Roininen

lassi.roininen@lut.fi

Manon Kok

m.kok-1@tudelft.nl

Roland Hostettler

roland.hostettler@angstrom.uu.se

Andreas Hauptmann

andreas.hauptmann@oulu.fi

1 Department of Electrical Engineering and Automation, Aalto University, 02150 Espoo, Finland

2 Department of Computational Engineering, LUT University, 53850 Lappeenranta, Finland

3 Delft Center for Systems and Control, Delft University of Technology, 2628 CD Delft, The Netherlands

4 Department of Electrical Engineering, Uppsala University, 75103 Uppsala, Sweden

5 Research Unit of Mathematical Sciences, University of Oulu, 90014 Oulu, Finland

6 Department of Computer Science, University College London, London WC1E 6BT, UK regular review procedure with necessary revisions before they were accepted. The papers included into the special issue are the following.

The paper Joint Estimation of Location and Scatter in Complex Elliptical Distributions: A Robust Semiparametric and Computationally Efficient R-estimator of the Shape Matrix studies the problem of joint estimation of the location vector and the shape matrix of a set of observations that are independent and identically complex-elliptic distributed. The paper studies the problem both theoretically and in terms of computational complexity, and presents a computationally efficient implementation of an estimator for shape matrices and a joint estimator of the location and the shape matrix. The paper Online Acoustic System Identification Exploiting Kalman Filtering and an Adaptive Impulse Response Subspace Model presents a novel algorithm for online estimation of acoustic impulse responses (AIR) which exploits prior knowledge about the AIR. The algorithm is shown to converge faster than the state-of-the-art and to have higher steady-state performance in scenarios suffering from high-level interfering noise. The paper Superquantile-based Learning: A Direct Approach Using Gradient-based Optimization describes the approach of supervised learning based on the superquantile risk measure and its implementation in an open source toolbox based on scikit-learn. The performance of the resulting gradient-based optimization method is illustrated in training models for several common benchmark problems such as classification of handwritten digits. Paper Multivariate Multifractal Texture DCGAN Synthesis: How Well Does It Work? How Does One Know? aims to propose a methodology that permits to quantify the quality of Deep Learning synthesized images.

Acoustic scene classification based on principal subspace projection is presented in the paper Unsupervised Domain Adaptation via Principal Subspace Projection for Acoustic Scene Classification. The proposed method addresses the challenge of generalization in soundscape classification under varying acoustic conditions and using different recording devices. This is achieved by first standardizing the spectro-temporal features of the audio signal, followed 
by projection onto the previously learned principal components. The resulting method is evaluated on two benchmark datasets, the TUT Urban Acoustic Scenes 2018 and the TAU Urban Acoustics Scenes 2020 dataset. The paper Multinomial Sampling of Latent Variables for Hierarchical Change-point Detection present a Bayesian approach for change-point detection. The proposed approach is based on a hierarchical latent variable model and a detection method based on multinomial sampling. The paper Fixing Bias in Reconstruction-based Anomaly Detection with Lipschitz.
Discriminators deals with anomaly detection and avoiding intrinsic biases by proposing a Lipschitz anomaly discriminator (LAD). Finally, the paper Compute and Memory Effcient Universal Sound Source Separation provides a family of efficient neural network architectures for general purpose audio source separation while focusing on multiple computational aspects that hinder the application of neural networks in real-world scenarios. 